\title{
Análise da consistência interna e fatorial confirmatório do IMPRAFE-126 com praticantes de atividades físicas gaúchos
}

\author{
Marcos Alencar Abaide Balbinotti - Universidade de Sherbrooke ${ }^{1}$ \\ Marcus Levi Lopes Barbosa - Universidade Federal do Rio Grande do Sul
}

\begin{abstract}
Resumo
Neste estudo, a motivação é entendida à luz da teoria da Autodeterminação. O objetivo deste estudo é verificar os índices de consistência interna e fatorial confirmatório do IMPRAFE-126. Utilizou-se uma amostra de 1.377 sujeitos, gaúchos, de ambos os sexos e com idades variando de 13 a 83 anos. Os resultados dos índices alfa de Cronbach (superiores a 0,89) foram satisfatórios. A adequação do modelo em seis dimensões foi testada e a validade confirmatória foi assumida para a amostra geral $\left(\chi^{2} / \mathrm{gl}=2,520\right.$; GFI=0,859; AGFI=0,854; RMSEA=0,065) e para os sexos masculino $\left(\chi^{2} / \mathrm{gl}=3,905 ; \mathrm{GFI}=0,885\right.$; AGFI=0,881; RMSEA=0,066) e feminino $\left(\chi^{2} / \mathrm{gl}=4,337\right.$; GFI=0,840; AGFI=0,831; RMSEA=0,068). Esses resultados indicam que o IMPRAFE-126 é um instrumento promissor e que pode ser oportunamente utilizado por psicólogos do esporte ou educadores físicos, aqueles particularmente interessados em avaliar os níveis de motivação de atletas ou praticantes de atividade física e esporte em geral. Entretanto, outros estudos de validade, fidedignidade e de normas devem ser conduzidos a fim de poder-se publicálos em um futuro próximo.

Palavras-chave: Motivação; Validação; Atividade física, IMPRAFE-126.
\end{abstract}

\section{Reliability and confirmatory factorial analysis of the IMPRAFE-126 with gauchos' practitioners of physical activities}

\begin{abstract}
In this study, motivation is understood in the context of Self-determination theory. This study aims to verify the index of reliability and confirmatory factorial validity of the IMPRAFE-126. A sample of 1.377 gauchos' practitioners of physical activities, both sexes with ages between 13 and 83 years old, was used. The results of Cronbach's alpha index (.89 to .94) had been satisfactory. The adequacy to the six-dimension model was tested and the construct confirmatory validity for the general sample was assumed $\left(\chi^{2} / \mathrm{gl}=2,520\right.$; GFI $=0,859$; AGFI=0,854; RMSEA=0,065), as so as for both sexes (masculine: $\chi^{2} / \mathrm{gl}=3,905$; GFI=0,885; AGFI=0,881; RMSEA=0,066; feminine: $\chi^{2} / \mathrm{gl}=4,337$; GFI=0,840; AGFI=0,831; RMSEA=0,068). These results indicate the IMPRAFE-126 is a promising tool that can be used by sports psychologists or personal trainers particularly interested in evaluate motivation levels of athletes or general practitioners of physical activities. New studies of validation, reliability and norms can be particularly helpful supposing a near future release.

Keywords: Motivation; Validation; Physical activity, IMPRAFE-126.
\end{abstract}

\section{Introdução}

Este artigo explora dados colhidos em academias, escolas e clubes esportivos do Rio Grande do Sul. Sua finalidade é verificar dois conjuntos importantes de propriedades psicométricas (estudos de consistência interna e fatorial confirmatório) de uma nova medida da motivação: o Inventário de Motivação à Prática Regular de Atividade Física e/ou Esporte (Balbinotti, 2004). Ainda, pretende-se discutir possíveis semelhanças e diferenças em alguns dados obtidos com esta amostra e a de outros estudos. Para melhor responder a estes objetivos, apresentam-se, inicialmente, aspectos referentes ao plano teórico relativo à motivação e, em seguida, aqueles referentes ao plano empírico (também relativo a este mesmo conceito). Finalmente, após a apresentação do método (procedimentos, sujeitos e instrumentos), são apresentados os resultados conforme os princípios métricos norteadores comumente aceitos na literatura especializada. As conclusões têm origem neste processo.

\section{A motivação no contexto da Teoria da Autodeterminação de Deci e Ryan}

Sabe-se que a prática de atividade física regular traz aos seus adeptos um grande número de benefícios em um amplo espectro de domínios que, em um bom

\footnotetext{
${ }^{1}$ Endereço para correspondência:

7 de l'Artiste - Kirkland - Québec - Canadá - H9J 4B6

E-mail: mbalbinotti@hotmail.com
} 
número de vezes, resulta em uma conseqüente melhora na qualidade de vida de seus praticantes (Paffenbarger, Hyde \& Wing, 1990; Wankel, 1993). Ainda assim, nem sempre as pessoas adotam a prática de atividade física regular por esse motivo; na verdade muitos dos possíveis motivos pelos quais uma pessoa adota a prática regular de atividade física e/ou esporte parecem estar pouco relacionados a este benefício (Gaya \& Cardoso, 1998). Conhecer os motivos pelos quais um sujeito possa vir a praticar uma determinada atividade física pode, quando adequadamente utilizado, aumentar as possibilidades de ingresso e permanência de indivíduos nesta prática (Gould, Udry, Tuffey \& Loehr, 1996; Ryan, Frederick, Lepes, Rubio \& Sheldon, 1997).

Segundo diversos autores (Anastasi \& Urbina, 2000; Balbinotti, 1994, 2001, 2005; Cunha, 1993, 2000; Gonzalez, 1992, 1997), a forma mais objetiva e sistemática de acessar este tipo de informação é com o uso de questionários, inventários e/ou escalas métricas de construtos psicossociais. O Inventário de Motivação para a Prática Regular de Atividade Física e/ou Esporte (Balbinotti, 2004) é um instrumento que viabiliza o acesso a essa informação. Sua construção baseou-se nos pressupostos da Teoria da Autodeterminação (Deci \& Ryan, 1985; Ryan \& Deci, 2000a).

Sistematizada por Deci e Ryan (1985, 2000a), a Teoria da Autodeterminação (Self-Determination Theory) é amplamente aceita e utilizada em diversas áreas do conhecimento acadêmico: educação (Deci \& Ryan, 2002; Reeve, Deci \& Ryan, 2004; Ryan \& Lynch, 2003), saúde (Sheldon, Williams \& Joiner, 2003; Williams, 2002; Williams, Deci \& Ryan, 1998), administração de empresas (Deci e cols., 2001; Gagné \& Deci, 2005; Vansteenkiste, Lens, Dewitte, Witte \& Deci, 2004), ambientalismo (Koestner, Losier, Vallerand \& Carducci, 2001; Pelletier, 2002; Pelletier, Dion, Tuson \& Green-Demers, 1999; Villacorta, Koestner \& Lekes, 2003), religião (Baard \& Aridas, 2001; Neyrinck, Lens \& Vansteenkiste, 2005; Ryan, Rigby \& King, 1993; Strahan \& Craig, 1995), política (Koestner e cols., 1996; Losier \& Koestner, 1999; Losier, Perreault, Koestner \& Vallerand, 2001), entre outros, inclusive no esporte e atividade física (Deci \& Olson, 1989; Frederick \& Ryan, 1995; Vallerand \& Losier, 1999). Esta teoria preconiza que um sujeito pode ser motivado em diferentes níveis (intrínseca ou extrinsecamente) ou, ainda, estar desmotivado à prática de qualquer atividade.

Quando intrinsecamente motivado, o sujeito ingressa na atividade por vontade própria, diga-se, pelo prazer e satisfação do processo de conhecê-la, explorá-la, aprofundá-la. Comportamentos intrinsecamente motivados são comumente associados com bem-estar psicológico, interesse, alegria e persistência (Ryan \& Deci, 2000b). A motivação tem sido subdividida em três tipos: para saber, para realizar e para experiência. A intrínseca para saber ocorre quando se executa uma atividade para satisfazer uma curiosidade, ao mesmo tempo em que se aprende tal atividade. A intrínseca para realizar ocorre quando um indivíduo realiza uma atividade pelo prazer de executá-la. A motivação intrínseca para experiência ocorre quando um indivíduo freqüenta uma atividade para experienciar as situações estimulantes, aquelas inerentes à tarefa (Brière e cols., 1995).

Já a motivação extrínseca, segundo Ryan e Deci (2000a), ocorre quando uma atividade é motivada por expectativas de resultados ou contingências não inerentes à própria atividade. Entretanto, esses motivos podem variar grandemente em relação ao seu grau de autonomia, criando, basicamente, três categorias dessa motivação: a) aquela de regulação externa: quando o comportamento é regulado por premiações materiais ou medo de conseqüências negativas, como críticas sociais (este tipo de motivação pode ser observado no âmbito esportivo quando o treinador impõe penas aos atletas se não realizarem as tarefas propostas); b) aquela de regulação interiorizada: quando o comportamento é regulado por uma fonte de motivação que, embora inicialmente externa, é internalizada, tais como aqueles realizados por pressões internas como a culpa, ou como a necessidade de ser aceito (este comportamento pode ser visto quando alguém realiza uma atividade por "descargo de consciência"); c) aquela de regulação identificada: quando um sujeito realiza uma tarefa (ou comportamento) que não escolheu, mas que é considerada importante (o sujeito reconhece a importância da tarefa) e faz, mesmo que não lhe seja interessante. Este tipo de comportamento é visualizado, por exemplo, no diálogo de um atleta que diz que aulas de alongamento são importantes porque seu treinador disse, e mesmo não gostando de executar ele o realiza.

Ryan e Deci (2000a), também citam a desmotivação (amotivation), construção motivacional percebida em indivíduos que ainda não estão adequadamente aptos a identificar um bom motivo para realizar alguma atividade física. Segundo esses indivíduos, tal atividade não lhes trará nenhum benefício ou não conseguirão realizá-la de modo satisfatório (Brière e cols., 1995).

Entretanto, convém serem feitas algumas ressalvas. Petherick e Weigand (2002) sugerem que a simples divisão entre motivação intrínseca e extrínseca pode gerar uma dicotomia simplista entre as duas. Também é necessário que se diga que ser motivado extrinsecamente não corresponde a um comportamento negativo. De acordo com Ryan e seus colaboradores (1997), os motivos extrínsecos podem possuir um grande grau de autonomia. Porém, motivos intrínsecos possuem caráter fundamentalmente autodeterminável.

Psico-USF, v. 13, n. 1, p. 1-12, jan./jun. 2008 
Por exemplo, um sujeito, logo após sofrer uma ameaça de enfarto, tem uma longa conversa com o médico, que explica detalhadamente a importância de mudanças nos seus hábitos de vida como forma de diminuir as chances de novas intercorrências. Disposto a diminuir os riscos a que está exposto, o sujeito passa a seguir rigorosamente as recomendações médicas na adoção de um estilo de vida mais saudável. $\mathrm{Na}$ situação descrita, a motivação do sujeito, embora seja extrínseca, é suficientemente intensa para garantir a manutenção do comportamento. Este tipo de situação é um típico exemplo de motivação extrínseca do tipo regulação identificada, onde ouve uma avaliação e endosso dos objetivos da atividade por parte do sujeito (Ryan \& Deci, 2000a).

\section{Medidas da motivacão à prática de atividade física}

As medidas são fundamentais no estudo da motivação. Diferentes instrumentos têm sido usados para medir este construto. No âmbito internacional, um importante instrumento foi desenvolvido por Gill e colaboradores (1983) com a construção do Participation Motivation Inventory (PMI-30). O PMI-30 é um instrumento composto por 30 itens que descrevem motivos para participar de um esporte, respondido em uma escala de tipo Likert em três pontos, indo de "nada importante" (1) a "muito importante" (3). O PMI-30 mede oito dimensões da motivação (auto-realização/ status, $\alpha=0,76$; prática em grupo, $\alpha=0,78$; forma física, $\alpha=0,75$; gasto de energia, $\alpha=0,65$; razões sociais, $\alpha=0,49$; desenvolver habilidades, $\alpha=0,44$; relações amistosas, $\alpha=0,30$; busca de distração, $\alpha=0,23)$. Embora este inventário apresente consistência interna insatisfatória $(\alpha<0,70)$ em cinco das suas oito dimensões, ele tem sido largamente utilizado em vários estudos nos EUA (Brodkin \& Weiss, 1990; Gould, Feltz \& Weiss, 1985; Klint \& Weiss, 1987).

Outro importante instrumento é o Intrinsic Motivation Inventory (IMI). Elaborado por Ryan em 1982, foi inicialmente utilizado por ele e seus colaboradores (Plant \& Ryan, 1985; Ryan e cols., 1983), entretanto, pouco tempo depois sofreu modificações e teve as suas qualidades métricas melhoradas por McAuley, Duncan e Tammen (1989). Trata-se de um instrumento composto por quatro subescalas (Interesse-envolvimento, $\alpha=0,78$; Percepção de competência, $\alpha=0,80$; Esforçoimportância, $\alpha=0,84$; Pressão-tensão, $\alpha=0,68$ ) com um total de 23 itens respondidos em uma escala de tipo Likert em sete pontos, indo de "concordo plenamente" a "discordo plenamente". Esta escala tem sido utilizada em diferentes estudos (Hassandra, Goudas \& Chroni, 2003, Kavussanu \& Roberts, 1996, Newton \& Duda, 1999) e a sua validade foi novamente discutida e confirmada em 1995 (Duda, Chi, Newton, Walling \& Catley, 1995).

Outra escala elaborada para a avaliação da motivação é o Exercise Motivation Inventory (EMI), elaborado por Markland e Hardy (1993). Não diferente dos outros, sua escala foi amplamente revisada e absorveu varias críticas que resultaram em uma nova versão, o EMI - 2 (Markland \& Ingledew, 1997). A segunda versão do EMI foi validada por meio de cinco análises fatoriais confirmatórias. Cada uma delas avaliou a validade de uma das cinco áreas cobertas pelo teste (motivos psicológicos, motivos interpessoais, motivo de saúde, motivos relacionados ao corpo e motivo de forma física), que se subdividem em 14 fatores: afiliação $(\alpha=0,91)$, aparência (aparência física; $\alpha=0,86$ ), desafio (desafio pessoal; $\alpha=0,86)$, competição $(\alpha=0,95)$, diversão (na atividade por si mesma; $\alpha=0,90$ ), pressão saúde (pressões provenientes de uma recomendação médica ou por uma condição médica específica; $\alpha=0,69$ ), prevenção saúde (prevenir problemas de saúde em geral; $\alpha=0,90)$, agilidade $(\alpha=0,90)$, promoção de saúde (promoção de bem-estar; $\alpha=0,88$ ), revitalização (senti-se bem após o exercício; $\alpha=0,83$ ), reconhecimento social $(\alpha=0,88)$, força e resistência $(\alpha=0,86)$, controle do estresse $(\alpha=0,92)$ e controle do peso $(\alpha=0,91)$. Os 51 itens respondidos em uma escala de tipo Likert em seis pontos, vão de "absolutamente falso para mim" (0) a "muito verdadeiro para mim" (5). Esta escala tem sido usada nos EUA (Ingledew, Markland \& Medley, 1998; Ingledew \& Sullivan, 2002) e fora dele (Capdevila, 2000; Capdevila, Niñerola \& Pintanel, 2004).

Existe ainda, na verdade, um grande número de escalas, tais como a Sport Motivation Scales (SMS) (Butt, 1987), com cinco escalas de cinco itens cada, que medem agressão, conflito, competência, competição e cooperação, a L'Échelle de Motivation pour les Sports (ÉMS) (Brière e cols., 1995), com sete fatores que medem diferentes aspectos da motivação intrínseca, extrínseca e da desmotivação. Ainda existem escalas que medem dimensões específicas da motivação, como a Social Motivational Orientations Scale for Sport (SMOSS) (Allen, 2003); entretanto, parecem ser escalas de menor impacto na literatura internacional (em virtude do número reduzido de artigos encontrados nos indexadores disponíveis).

No âmbito nacional, alguns estudos têm procurado desenvolver escalas adequadas à cultura local (que não sejam simples traduções das escalas internacionais) e que tenham propriedades métricas que recomendem sua utilização. Um esforço neste sentido deu origem ao Inventário de Motivação para a Prática Desportiva (IMPD) (Gaya \& Cardoso, 1998). O IMPD é uma escala com 19 itens distribuídos em três dimensões: 
competência esportiva $(\alpha=0,61)$, amizade-lazer $(\alpha=0,64)$ e saúde $(\alpha=0,64)$.

Outra escala recentemente construída no contexto nacional é o Inventário de Motivação para a Prática Regular de Atividade Física e/ou Esporte (IMPRAFE-126) (Balbinotti, 2004). Trata-se de um inventário que pretende avaliar seis das possíveis dimensões associadas à prática regular de atividade física e/ou esporte. São 120 itens agrupados 6 a 6, seguindo a seqüência das dimensões mais freqüentemente mencionadas na literatura (Barbosa, 2006), a saber: controle do estresse (ex.: liberar tensões mentais), saúde (ex.: manter a forma física), sociabilidade (ex.: estar com amigos), competitividade (ex.: vencer competições), estética (ex.: manter bom aspecto) e prazer (ex.: meu próprio prazer).

As respostas aos itens deste inventário são dadas conforme uma escala bidirecional, de tipo Likert, graduada em cinco pontos, indo de "isto me motiva pouquíssimo" (1) a "isto me motiva muitíssimo" (5). O inventário conta ainda com uma escala de verificação que permite a avaliação do nível de atenção do sujeito durante a aplicação. Seis itens, um de cada dimensão, tomados aleatoriamente, são repetidos no final do inventário. A medida da validade da aplicação é obtida através da análise das diferenças entre as respostas. São precisamente as qualidades métricas (validade fatorial confirmatória e consistência interna) desta escala que se avaliarão neste estudo.

\section{Questão central desta pesquisa}

Partindo-se dos planos teóricos e empíricos apresentados anteriormente, foi possível formular a seguinte questão de pesquisa: a partir de dados colhidos na população de praticantes de atividades físicas gaúchos, poder-se-ão encontrar índices de validade confirmatória e fidedignidade adequados para o uso do IMPRAFE-126? Para bem responder a esta questão, foram empregados procedimentos metodológicos, éticos e estatísticos. Esses procedimentos serão apresentados a seguir.

\section{Método}

\section{Procedimentos, Sujeitos e Instrumentos}

Os praticantes de atividade física regular foram contatados em diferentes contextos sociais onde atividades físicas regulares são realizadas (clubes, academias, escolas, etc.). No contato inicial, o pesquisador se identificou, explicou o tema e o objetivo da pesquisa. Quando se mostrou necessário, foi agendada uma visita onde outras explicações eram apresentadas, sempre a pedido do praticante. Após, os convidados, especificamente aqueles que aceitaram participar, assinaram o consentimento informado. Por fim, considerando que não era exigida a identificação por nome dos participantes, os praticantes foram assegurados da confidencialidade de suas respostas.

Assim, um total de 1.377 praticantes de atividade física regular, de ambos os sexos, e com idades que variaram de 13 a 83 anos, responderam o IMPRAFE-126. Destaca-se que esta amostra foi escolhida pelos critérios de disponibilidade e acessibilidade (Maguire \& Rogers, 1989). Com o intuito de melhor informar o leitor, a Tabela 1 apresenta a freqüência de ocorrência dos sujeitos nos grupos de acordo com sexo e idade.

Tabela 1 - Freqüência dos sujeitos por subgrupos da amostra

\begin{tabular}{|c|c|c|c|c|c|c|c|}
\hline \multicolumn{2}{|c|}{ Variáveis } & \multicolumn{2}{|c|}{ Sexo } & \multicolumn{4}{|c|}{ Grupos de idade (em anos) } \\
\hline & & M & $\mathrm{F}$ & 13 a 20 & 21 a 40 & 41 a 65 & 66 a 83 \\
\hline \multirow{2}{*}{ Sexo } & $\mathrm{M}$ & 662 & - & - & - & - & - \\
\hline & $\mathrm{F}$ & - & 715 & - & - & - & - \\
\hline \multirow{4}{*}{$\begin{array}{l}\text { Grupos de } \\
\text { idade } \\
\text { (em anos) }\end{array}$} & 13 a 20 & 428 & 339 & 767 & - & - & - \\
\hline & 21 a 40 & 202 & 176 & - & 378 & - & - \\
\hline & 41 a 65 & 30 & 152 & - & - & 182 & - \\
\hline & 66 a 83 & 2 & 48 & - & - & - & 50 \\
\hline
\end{tabular}

A informação sobre a idade dos sujeitos foi apresentada de forma resumida, agrupada conforme sugerido por Papalia, Olds e Feldman (2006), a saber: de 13 a 20 anos (adolescente, 55,7\%), de 21 a 40 anos (jovem adulto, 27,5\%), de 41 a 65 anos (meia-idade, 13,2\%), e de 66 a 83 (terceira-idade, 3,6\%). Destaca-se que esta amostra é composta por $48,1 \%$ de sujeitos do sexo masculino e $51,9 \%$ do sexo feminino.

Quanto aos instrumentos, foram utilizados dois: um Questionário Biossóciodemográfico Simples (QBSDS), apenas para controle das variáveis sexo e idade, e o Inventário de Motivação à Prática Regular de Atividade 
Física e/ou Esporte (IMPRAFE-126), que já foi anteriormente descrito.

\section{Resultados e discussão}

Para responder adequadamente à questão central desta pesquisa, procedeu-se à exploração dos escores obtidos pelo IMPRAFE-126, segundo princípios norteadores comumente aceitos na literatura especializada (Angers, 1992; Balbinotti, 2005; Bisquera, 1987; Bryman \& Cramer, 1999; Cronbach \& Meehl, 1955; Dassa, 1999; Nunnally, 1978; Pestana \& Gageiro, 2003; Reis, 2001; Trudel \& Antonius, 1991; Vallerand, 1989). Caminho feito, apresentam-se, sucessiva e sistematicamente, os resultados do índice alfa de Cronbach (para estudo da consistência interna da escala) e da análise fatorial confirmatória (para o estudo da validade de construto do inventário).

\section{Indice alfa de Cronbach}

Antes de se chegar às análises propriamente ditas dos índices alfa de Cronbach obtidos por esta amostra, foram conduzidas estatísticas preliminares que fundamentam este índice de consistência interna. Destaca-se que a apresentação formal destas estatísticas, neste estudo, tem por objetivo único demonstrar a confiabilidade dos valores das médias observadas; pois estas, por poderem sofrer efeitos negativos pela presença de casos extremos (Pestana e Gageiro, 2003), poderiam não ser representativas dos comportamentos inventariados, diminuindo o valor das conclusões (Balbinotti, 2005).

Assim, destaca-se que as médias encontradas para cada um dos 120 itens, estudados individualmente, variaram entre 1,71 e 4,37 (=3,25); com desvios padrão (DP) associados variando entre 0,95 a 1,57. Interpretam-se esses resultados preliminares como sendo satisfatórios, pois não houve uma aderência predominante (seja positiva ou negativa) a nenhum dos itens isolados; diga-se, itens com médias iguais ou extremamente próximas aos valores extremos (1 ou 5). Destaca-se, ainda, que a variabilidade dos resultados foi restrita, denotando-se, assim, certa homogeneidade na dispersão avaliada, independente do item individualmente estudado.

Quanto às médias encontradas nas dimensões, estas variaram de 56,23 a 74,43 (sendo que a média esperada era de 60 pontos), com desvios padrão (DP) associados variando entre 14,66 a 19,33. Considerando a escala de resposta, os valores esperados por cada uma das seis dimensões poderiam variar de 20 a 100 pontos. Efetivamente, foram observadas variações de 20 a 100 pontos em todas as subescalas, denota-se que os valores extremos observados são idênticos àqueles esperados.
Finalmente, os índices das correlações itemdimensão apresentaram-se bastante satisfatórios em todas as dimensões: controle do estresse (variando de $r=0,36$ a $r=0,72$ ), saúde (variando de $r=0,37$ a $r=0,68$ ), sociabilidade (variando de $r=0,32$ a $r=0,78$ ), competitividade (variando de $r=0,38$ a $r=0,80$ ), estética (variando de $r=0,35$ a $r=0,73$ ) e prazer (variando de $r=0,35$ a $r=0,66$ ). A partir dessas importantes constatações, que garantem a base do cálculo de consistência interna, o coeficiente alfa de Cronbach pode ser calculado.

O coeficiente alfa foi obtido a partir do SPSS (versão 13,0 para Windows), verificando-se a consistência interna do IMPRAFE-126. Foi utilizado o modelo proposto por Cronbach e seus colaboradores (Cronbach, 1951, 1988, 1989, 1996; Cronbach \& Meehl, 1955; Cronbach, Rajaratnam \& Gleser, 1963). Basicamente, eles consideram que, por um lado, cada item deve estar satisfatoriamente correlacionado com sua própria dimensão (ou fator) e, por outro lado, não devem existir correlações negativas entre um item e a escala total. Garson (2005), Pestana e Gageiro (2003), Nunnally (1978) e Taylor e colaboradores (2003) recomendam que a adequação e a satisfatoriedade do modelo alfa sejam testadas usando-se os seguintes critérios: índices alfa superiores a 0,80 são considerados desejáveis; índices superiores a 0,70 são considerados recomendados; índices superiores a 0,60 devem ser aceitos apenas para uso em pesquisa (desaconselhável o uso clínico). Sendo assim, qualquer resultado superior a 0,60 pode ser interpretado como uma consistência interna satisfatória, no enquadramento desta pesquisa.

Com base nesses dados preliminares apresentados, pode-se indicar o coeficiente alfa para as dimensões do inventário em estudo, a saber: controle do estresse $(\alpha=0,92)$, saúde $(\alpha=0,90)$, sociabilidade $(\alpha=0,93)$, competitividade $(\alpha=0,94)$, estética $(\alpha=0,92)$ e prazer $(\alpha=0,89)$. Estes resultados indicam índices desejáveis de consistência interna para o IMPRAFE-126. Assim, podese dizer que os itens contitutivos das seis dimensões do IMPRAFE-126 apresentam precisão em suas medidas (quando analisados em suas próprias dimensões).

$\mathrm{Na}$ verdade, o conjunto dos resultados relativos à consistência interna obtido para o IMPRAFE-126 é melhor do que aqueles observados em outros estudos tanto no âmbito internacional, onde os índices de consistência interna variaram, respectivamente, de 0,23 a 0,78 (Gill e cols., 1983), de 0,68 a 0,84 (McAuley e cols., 1989) e de 0,69 a 0,95 (Markland \& Hardy, 1993; Markland \& Ingledew, 1997), quanto no nacional (Gaya \& Cardoso, 1998), onde esses índices variaram de 0,61 a 0,64 . Sendo assim, o IMPRAFE-126 destaca-se pela satisfatoriedade de seus resultados relativos à consistência interna. 
A fim de saber se o modelo em seis dimensões é suficiente para avaliar o construto "motivação à prática regular de atividade física e/ou esporte", é indicado que se teste sua validade de construto com ajuda do modelo fatorial confirmatório (Anderson \& Gerbing, 1984).

\section{Análise fatorial confirmatória}

Antes de se chegar aos cálculos relativos à validade fatorial confirmatória propriamente dita, uma análise preliminar foi realizada a fim de verificar a adequação dos dados à análise. Trata-se precisamente da verificação da existência de casos aberrantes (outliers), já que a inexistência desses casos é um pressuposto para esta análise (Balbinotti, 2005). Com o auxílio do "Gráfico de
Bigodes" (obtido com a ajuda do SPSS) foi possível descartar a presença desses casos e concluir tratar-se de dados adequados ao procedimento da análise fatorial confirmatória.

Sendo assim, o modelo de seis fatores do IMPRAFE-126 (ver Figura 1) foi testado a partir do pacote AMOS (versão 4.0 para Windows), verificando-se sua adequabilidade (Berry, Poortinga, Segall \& Dasen, 1992). Foi usado o modelo proposto por Bagby, Taylor e Parker (1992), que considera que cada item deve aferir apenas um fator, diferenciando-se, portanto, do modelo fatorial exploratório (onde cada item apresenta saturações fatoriais nos diversos fatores com valores próprios superiores a 1).

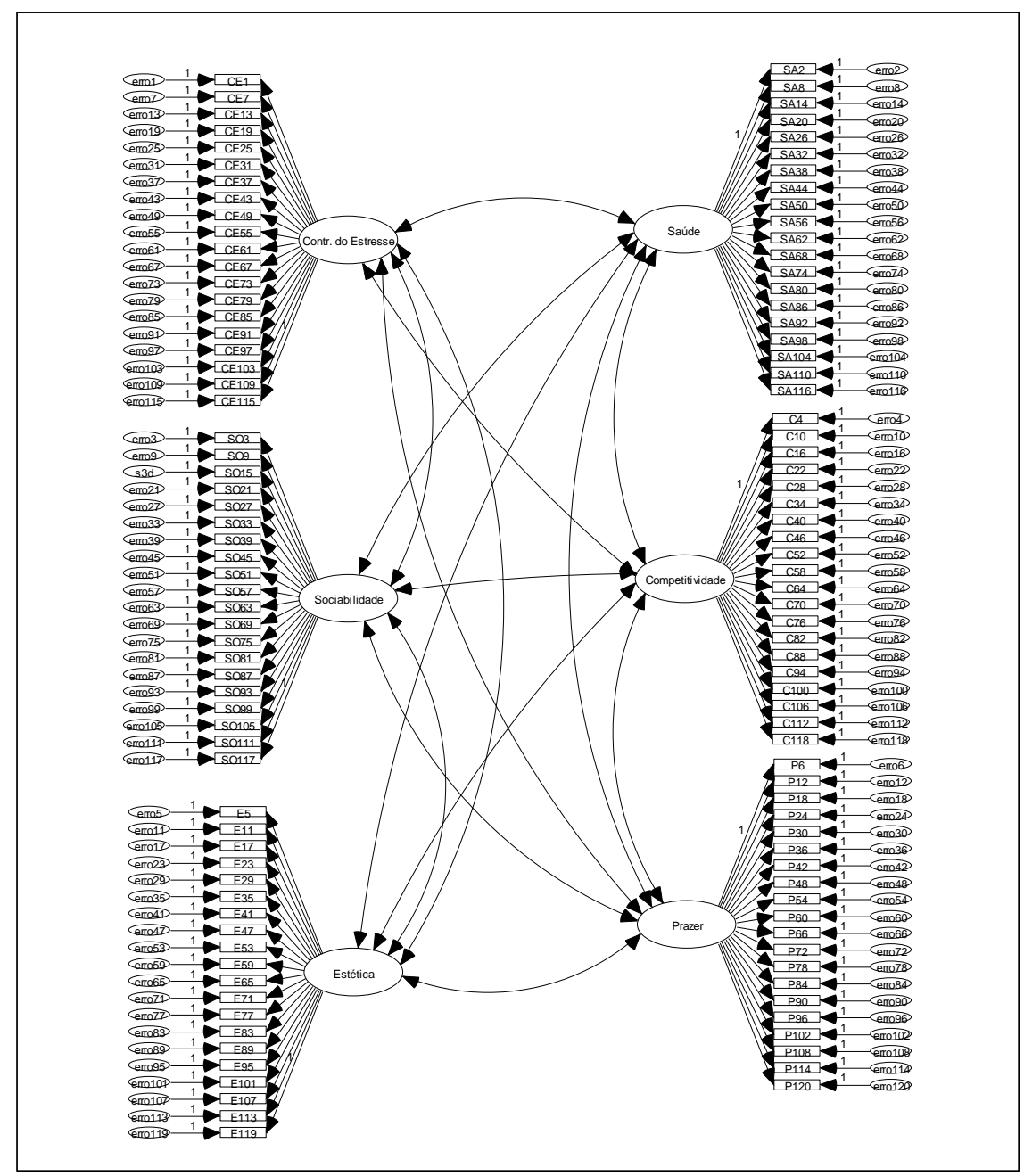

Figura 1 - Modelo teórico de avaliação da motivação à pratica regular de atividades físicas proposto por Balbinotti (2004)

Assim, partindo-se da hipotética associação entre as seis dimensões do construto avaliado para a amostra de praticantes de atividade física regular e/ou esporte, seguindo as recomendações de Cole (1987), Watkins
(1989) e Briggs e Cheek (1986), a adequação do modelo fatorial confirmatório foi testada usando os cinco seguintes critérios: qui-quadrado, razão entre quiquadrado e graus de liberdade, GFI (Goodness-of-fit Index), 
AGFI (Adjusted Goodness-of-Fit Index) e a raiz quadrada média residual (RMS).

Critérios múltiplos foram utilizados uma vez que cada índice apresenta diferentes forças e fraquezas na avaliação da adequação do modelo fatorial confirmatório (Taylor, Bagby \& Parker, 2003). São eles: o teste Qui-quadrado deve ser não-significativo; a razão entre qui-quadrado e graus de liberdade deve ser menor que 5 (em valores nominais) ou, desejavelmente, menor que 2; o GFI deve apresentar um índice superior ou igual a 0,85 ; o AGFI deve apresentar um índice superior ou igual a 0,80 ; e, finalmente, o RMS deve apresentar um índice inferior ou igual a 0,10 (Anderson \& Gerbing, 1984; Balbinotti, 2005; Cole, 1987; Marsh, Balla \& McDonald,1988).

A amostra avaliada apresentou (ver Tabela 2) um qui-quadrado significativo $(p<0,001)$ e a razão entre o qui-quadrado e graus de liberdade insatisfatório $\left(\chi^{2} / \mathrm{gl}>5\right)$. Resultados insatisfatórios, como esses, são tipicamente encontrados em grandes amostras. Por essa razão, alguns autores (Cole, 1987; Marsh e cols., 1988) têm descartado, especialmente o qui-quadrado, de suas análises, pois se trata de uma estatística extremamente sensível ao número de sujeitos da amostra.

No que diz respeito à razão entre o quiquadrado e graus de liberdade, um procedimento simples foi conduzido com vistas a verificar se o resultado insatisfatório ocorreu, de fato, pela grande amostra utilizada na análise ou se, realmente, não há adequação dos dados ao modelo. Por meio do comando "Select Cases" do SPSS, foram aleatoriamente selecionados $20 \%$ dos casos da amostra inicialmente utilizada. A nova análise conduzida com estes casos mostrou resultado satisfatório $\left(\chi^{2} / g 1=2,52\right)$. Esse procedimento foi repetido por seis vezes; todos os resultados mostraram-se satisfatórios (variando de 2,34 a 2,76).

Tabela 2 - Índices de validade fatorial confirmatória para a amostra geral e subgrupos da amostra

\begin{tabular}{cccccccc}
\hline \multirow{2}{*}{ Grupos } & \multicolumn{7}{c}{ Índices de validade fatorial confirmatória } \\
& $\chi^{2}$ & $\mathrm{gl}$ & Sig. & $\chi^{2} / \mathrm{gl}$ & GFI & AGFI & RMS \\
\hline Geral & 48270,509 & 7005 & $<0,001$ & 6,890 & 0,859 & 0,854 & 0,065 \\
Masculino & 27354,735 & 7005 & $<0,001$ & 3,905 & 0,885 & 0,881 & 0,066 \\
Feminino & 30387,285 & 7005 & $<0,001$ & 4,337 & 0,840 & 0,831 & 0,068 \\
\hline
\end{tabular}

As demais importantes medidas de adequação ao modelo estão em acordo com os critérios padrões, o que permite assumir a validade de construto do inventário. Dessa forma, uma vez que a validade de construto, pelo viés da análise fatorial confirmatória do inventário, foi estabelecida para a amostra total, análises independentes por sexo foram conduzidas.

Sendo assim, obteve-se um qui-quadrado significativo para os dois sexos $(p<0,001)$, como já era esperado (em função do $n$ amostral). As demais importantes medidas de adequação ao modelo apresentaram-se em acordo com os critérios padrão (ver Tabela 2), o que permite assumir a validade do inventário para os dois sexos.

De forma geral, estudos de validade de medidas da motivação têm usado o modelo fatorial exploratório (Butt, 1987; Gill e cols., 1983; Markland \& Hardy, 1993; McAuley e cols., 1989), que é um importante método de avaliação da validade de construto de questionários, inventários e/ou escalas (Dassa, 1999; Pestana \& Gageiro, 2003). Entretanto, foram encontrados alguns pequenos problemas (e outros não tão pequenos) em alguns desses estudos. Uma análise pormenorizada dos estudos citados neste manuscrito indica que, em alguns casos (Gaya \& Cardoso, 1998; Gill e cols., 1983; Ryan e cols., 1997), os autores incluíram diversos itens complexos (itens com saturação fatorial superior a 0,30 em mais de um fator) ou com saturação fatorial insuficiente (itens com saturação fatorial inferior a 0,30 ), o que não é recomendável (Dassa, 1999; Pestana \& Gageiro, 2003), principalmente considerando o $n$ amostral. Tais aspectos reduzem a confiança nos resultados (Balbinotti, 2005).

O estudo realizado por Markland e Ingledew (1997) utilizou o modelo fatorial confirmatório e preocupou-se em verificar a validade do modelo de medida da motivação para a amostra total e para os sexos masculino e feminino (o que é raro). Entretanto, cabe salientar que, neste estudo, os autores optaram por não testar todo o modelo de uma vez. Ao invés disso, os autores optaram por reagrupar as 14 dimensões do inventário em cinco grupos de dimensões (agrupadas por afinidade de conteúdo) e testar a validade de cada um desses grupos, independentemente. Com esta estratégia, os autores sabiamente reduziram os riscos de ver o seu modelo rejeitado. Problemas e opções metodológicas como estas reforçam a importância dos resultados encontrados com o IMPRAFE-126. O rigor metodológico e a satisfatoriedade dos resultados obtidos neste estudo colocam o instrumento avaliado em posição diferenciada. 


\section{Conclusões}

Este estudo teve por objetivo verificar importantes qualidades métricas (alfa de Cronbach e modelo fatorial confirmatório) do IMPRAFE-126 com praticantes de atividade física gaúchos. Para poder-se responder adequadamente à questão central desta pesquisa, inicialmente, postulou-se, teoricamente, que um sujeito pode ser motivado em diferentes níveis (intrínseca ou extrinsecamente), ou ainda, desmotivado durante a prática de uma atividade qualquer. Após, apresentaram-se os instrumentos de medida utilizados nos âmbitos internacional e nacional, para avaliar a motivação. Esses tópicos formaram a base para a elaboração da questão central desta pesquisa.

Quanto às conclusões relativas ao cálculo alfa de Cronbach, verificou-se que o IMPRAFE-126 é consistente, o que significa que as correlações interitens foram satisfatórias quando consideram-se os praticantes de atividades físicas que compuseram a amostra deste estudo. Destaca-se que não houve correlações negativas entre os pares de itens das dimensões. No mais, destaca-se que o conjunto de índices de consistência interna encontrado para as dimensões do IMPRAFE-126 ( $\alpha$ entre 0,89 e 0,94 ) é melhor do que aquele encontrado em outros estudos, com outros instrumentos. Quanto às conclusões relativas à análise fatorial confirmatória, verificou-se que o IMPRAFE-126 apresenta índices de validade fatorial confirmatória satisfatórios, tanto para a amostra geral quanto para os sexos (separadamente). Esses resultados permitiram assumir a validade de construto do modelo de avaliação da motivação proposto pelo IMPRAFE-126.

Antes de finalizar, cabe destacar que esses resultados podem ser particularmente úteis para psicólogos do esporte, personal trainers e outros profissionais interessados por assuntos relacionados à atividade física $\mathrm{e}$ exercício com adolescentes, jovens, adultos e idosos (de 13 a 83 anos), que visam avaliar (explorar) seus níveis de motivação à prática regular de atividade física e/ou esporte. Então, o IMPRAFE-126 apresenta-se como uma promissora forma de avaliação da motivação à prática regular de atividade física e/ou esporte, e as dimensões deste construto (controle de estresse, saúde, sociabilidade, competitividade, estética e prazer) parecem ser uma fonte importante de informação para esses profissionais, permitindo que eles entendam melhor como esses elementos se integram na dinâmica geral de funcionamento daqueles que se beneficiam.

Por fim, destacam-se algumas das importantes limitações desta pesquisa: não tratar-se de dados colhidos aleatoriamente e os praticantes de atividade física serem todos gaúchos. Novos estudos deveriam dar conta dessas limitações ao mesmo tempo em que testar outras importantes propriedades psicométricas do IMPRAFE-126 a fim de torná-lo publicável em um futuro próximo. Ressalta-se, ainda, a importância de novos estudos com amostras de populações específicas de praticantes de atividades físicas brasileiros (como aqueles com necessidades especiais, por exemplo).

\section{Referências}

Allen, J. B. (2003). Social motivation in youth sport. Journal of Sport \& Exercise Psychology, 25, 551-567.

Anastasi, A. \& Urbina, S. (2000). Testagem psicológica (7 $7^{\mathrm{a}} \mathrm{ed}$.). Porto Alegre: Artes Médicas.

Anderson, J. C. \& Gerbing, D. W. (1984). The effect of sampling error on convergence, improper solutions, and goodness-of-fit indices for maximum likelihood confirmatory factor analysis. Psychometrika, 49, 155-173.

Angers, M. (1992). Initiation pratique à la methodologie des sciences bumaines. Montreal: Les Éditions de la Chenelière.

Baard, P. P. \& Aridas, C. (2001). Motivating your church: How any leader can ignite intrinsic motivation and growth. Nova Iorque: Crossroads.

Bagby, R. M., Taylor, G. J. \& Parker, J. D. (1992). Reliability and validity of the 20-Item Toronto-Alexithymia-Scale. Pôster apresentado no encontro do $50^{\circ}$ Aniversário da American Psychosomatic Society. Nova Iorque.

Balbinotti, M. A. A. (1994). Significado social do conceito e da avaliação da inteligência: Perspectiva histórica e levantamento de opiniōes. Dissertação de Mestrado. Porto Alegre, RS: PUCRS - Programa de Mestrado em Psicologia Social e da Personalidade.

Balbinotti, M. A. A. (2001). Vers um modèle explicatif de la cristallisation des préférences professionnelles durant l'adolescence. Tese de Doutorado. Montreal, Canadá: Universidade de Montreal - Faculdade de Artes e Ciências, Programa de $\mathrm{PhD}$ em Psicologia.

Balbinotti, M. A. A. (2004). Inventário de motivação à prática regular de atividade física e/ou esporte. Porto Alegre: Universidade Federal do Rio Grande do Sul Laboratório de Psicologia do Esporte.

Balbinotti, M. A. A. (2005). Para se avaliar o que se espera: reflexões acerca da validade dos testes psicológicos. Aletheia, 1(21), 43-52.

Barbosa, M. L. L. (2006). Propriedades métricas do Inventário de Motivação para a Prática Regular de Atividade Física (IMPRAFE-126). Dissertação de Mestrado. Porto Alegre, RS: Universidade Federal do Rio Grande do Sul - Programa de Pós-Graduação em Ciências do Movimento Humano, Atividade Física e Performance. 
Berry J. W., Poortinga Y. H., Segall M. H. \& Dasen P. R. (1992). Cross-cultural psychology: research and applications. Cambridge (UK): Cambridge Univ. Press.

Bisqueira, R. (1987). Introducción a la estadística aplicada a la investigation educativa: um enfoque informático com los paquetes BMDP y SPSS. Barcelona: PPU.

Brière, N., Vallerand, R., Blais, M. \& Pelletier, L. (1995). Developpement et validation d'une mesure de motivation intrinséque, extrinséque et d'amotivation en contexte sportif: l'echelle de motivation dans les sports. International Journal of Sport Psychology, 26(4), 465-489.

Briggs S. R. \& Cheek J. M. (1986). The role of factor analysis in the development and evaluation of personality scales. Journal of Personality, 54, 6-48.

Bryman, A. \& Cramer, D. (1999). Quantitative data analysis: A guide for social scientists. Nova Iorque: Routledge.

Brodkin, P. \& Weiss, M. (1990). Developmental differences in motivation for participating in competitive swimming. Journal of Sport \& Exercise Psychology, 12, 248-263.

Butt, D. S. (1987). Psychology of sport: The behavior, motivation, personality, and performance of atbletes. Nova Iorque: Van Nostrand Reinhold.

Capdevila, L. (2000). Actividad fisica y estilo de vida saludable $\left(2^{\mathrm{a}}\right.$ ed.). Barcelona: Artyplan.

Capdevila, L., Niñerola, J. \& Pintanel, M. (2004). Motivación y actividad física: el autoinforme de motivos para la práctica de ejercicio físico (AMPEF). Revista de Psicología del Deporte, 13(1), 55-74.

Cole, D. A. (1987). Utility of confirmatory factor analysis in test validation research. Journal of Consulting and Clinical Psychology, 55, 584-594.

Cronbach, L. J. (1951). Coefficient alpha and the internal structure of tests. Psychometrika, 16, 297-334.

Cronbach, L. J. (1988). Initernal-consistency of tests: Analyses old and new. Psychometrika, 53, 63-70.

Cronbach, L. J. (1989). Construct validation after thirty years. Em R. L. Linn (Ed.). Intelligence: Measurement, theory and public policy - Proceedings of a symposium in honor of Lloyd G. Hamphreys. Chicago, IL: University of Chicago Press.

Cronbach, L. J. (1996). Fundamentos da testagem psicológica. Porto Alegre, RS: Artes Médicas.

Cronbach, L. J. \& Meehl, P. E. (1955). Construct validity in psychological tests. Psychological Bulletin, 52, 281-302.

Cronbach, L. J., Rajaratnam, N. \& Gleser, G. C. (1963). Tehory of generalizability: A liberalization of reliability theory. The British Journal of Statistical Psychology, 16(2), 137-163.
Cunha, J. A. (1993). Psicodiagnóstico-R (4a ed. rev.). Porto Alegre: Artes Médicas.

Cunha, J. A. (2000). Psicodiagnóstico-R ( $5^{\mathrm{a}}$ ed. rev.). Porto Alegre: Artes Médicas Sul.

Dassa, C. (1999). Analyse multidimensionnelle exploratoire et confirmative. Montreal: Univesité de Montreal.

Deci, E. L. \& Olson, B. C. (1989). Motivation and competition: Their role in sports. Em J. H. Goldstein (Ed.). Sports, games, and play ( $2^{\mathrm{a}}$ ed., pp. 83-110). Hillsdale, NJ: Erlbaum.

Deci, E. L. \& Ryan, R. M. (1985). Intrinsic motivation and selfdetermination in human behavior. Nova Iorque: Plenum.

Deci, E. L. \& Ryan, R. M. (2002). The paradox of achievement: The harder you push, the worse it gets. Em J. Aronson (Ed.). Improving academic achievement: Contributions of social psychology (pp. 59-85). Nova Iorque: Academic Press.

Deci, E. L., Ryan, R. M., Gagné, M., Leone, D. R., Usunov, J. \& Kornazheva, B. P. (2001). Need satisfaction, motivation, and well-being in the work organizations of a former Eastern Bloc country. Personality and Social Psychology Bulletin, 27, 930-942.

Duda, J., Chi, L., Newton, M. Walling, M. \& Catley, D. (1995). Task and ego orientation and intrinsic motivation in sport. International Journal of Sport Psychology, 26(1), 81-97.

Frederick, C. M. \& Ryan, R. M. (1995). Self-determination in sport: A review using cognitive evaluation theory. International Journal of Sport Psychology, 26, 5-23.

Gagné, M. \& Deci, E. L. (2005). Self-determination theory and work motivation. Journal of Organizational Behavior, 26, 331-362.

Garson, D. (2005). PA 765 Statnote: An online textbook. Obtido em 6 de março de 2005 do World Wide Web: http://www2.chass.ncsu.edu/garson/pa765/statnote.ht $\mathrm{m}$.

Gaya, A. \& Cardoso, M. (1998). Os fatores motivacionais para a prática desportiva e suas relações com o sexo, idade e níveis de desempenho desportivo. Revista Perfil, 2(2), 40-51.

Gill, D. L., Gross, J. B. \& Huddleston, S. (1983). Participation motivation in youth sports. International Joumal of Sport Psychology, 14, 1-14.

Gonzalez, M. P. (1992). La intervención educativa para el desarrollo de la carrera: análisis de las necesidades de desarrollo para la carrera de los estudiantes al finalizar la éducatión secundaria. Tese de Doutorado. Espanha: Universidade de Olviedo - Faculdade de Ciências da Educação, Programa de Doutorado em Ciências da Educação. 
Gonzalez, M. P. (1997). Career education needs of secondary school graduates from Asturias, Spain. Journal of Career Development, 23(3), 215-229.

Gould, D., Feltz, D. \& Weiss, M. (1985). Motives for patiticipating in competitive youth swimming. International Journal of Sport Psychology, 16, 126-140.

Gould, D., Udry, E., Tuffey, S. \& Loehr, J. (1996). Burnout in competitive junior tennis players: I. A quantitative psychological assessment. The Sport Psychologist, 10, 322340 .

Hassandra, M., Goudas, M. \& Chroni, S. (2003). Examining factors associated with intrinsic motivation in physical education: a qualitative approach. Psychology of Sport and Exercise, 4, 211-223.

Ingledew, D. K. \& Sullivan, G. (2002). Effects of body mass and body image on exercise motives in adolescence. Psychology of Sport and Exercise, 3, 323-338.

Ingledew, D. K. I., Markland, D. \& Medley, A. (1998). Exercise motives and stages of change. Journal of Health Psychology, 3, 477-489.

Kavussanu, M. \& Roberts, G. C. (1996). Motivation in physical activity contexts: The relationship of perceived motivational climate to intrinsic motivation and selfefficacy. Journal of Sport and Exercise Psychology, 18, 264-280.

Klint, K. A. \& Weiss, M. R. (1987). Perceived competence and motives for participation in youth sports: a test of Harter's competence motivation theory. Journal of Sport Psychology, 9, 55-65.

Koestner, R., Losier, G. F., Vallerand, R. J. \& Carducci, D. (1996). Identified and introjected forms of political internalization: Extending self-determination theory. Journal of Personality and Social Psychology, 70, 1025-1036.

Koestner, R., Houlford, N., Paquet, S. \& Knight, C. (2001), On the risks of recycling because of guilt: An examination of the consequences of introjection. Journal of Applied Social Psychology, 31, 2545-2560.

Losier, G. F. \& Koestner, R. (1999). Intrinsic versus identified regulation in distinct political campaigns: The consequences of following politics for pleasure versus personal meaningfulness. Personality and Social Psychology Bulletin, 25, 287-298.

Losier G. F., Perreault, S., Koestner, R. \& Vallerand, R. J. (2001). Examining individual differences in the internalization of political values: Validation of the selfdetermination scale of political motivation. Journal of Research in Personality, 35, 41-61.

Maguire, T. O. \& Rogers W. T. (1989). Proposed solutions for non randomness in educational research. Canadian Journal of Education, 14(2), 170-181.
Markland, D. \& Hardy, L. (1993). The Exercise Motivations Inventory: Preliminary development and validity of a measure of individuals' reasons for participation in regular physical exercise. Personality and Individual Differences, 15, 289-296.

Markland, D. \& Ingledew, D. K. (1997). The measurement of exercise motives: Factorial validity and invariance across gender of a revised Exercise Motivations Inventory. British journal of Health Psychology, 2, 361-376.

Marsh, H. W., Balla, J. R. \& McDonald, R. P. (1988). Goodness-of-fit indexes in confirmatory factor analisys: the effect of sample size. Psychological Bulletin, 103, 391-410.

McAuley, E., Duncan, T. A. \& Tammen, V. V. (1989). Psychometric properties of the Intrinsic Motivation Inventory in a competitive sport setting: A confirmatory factor analysis. Research Quarterly for Exercise and Sport, 60, 48-58.

Newton, M. \& Duda, J. (1999). The interaction of motivational climate, dispositional goal orientations, and perceived ability in predicting indices of motivation. International Journal of Sport Psychology, 30, 63-82.

Neyrinck, B., Lens, W. \& Vansteenkiste, M. (2005). Goals and regulations of religiosity: A motivational analysis. Em M. L. Maehr \& S. Karabenick (Eds.). Advances in motivation and achievement (pp. 77-106). Greenwich, Conn.: Jai Press Inc.

Nunnally, J. C. (1978). Psychometric Theory (2a ed.). Nova Iorque: McGraw-Hill.

Paffenbarger, R. S., Hyde, R. T. \& Wing, A. L. (1990). Physical activity and physical fitness as determinants of health and longevity. Em C. Bouchard, R. J. Shephard, T. Stephens, J. R. Sutton \& B. D. McPherson (Eds.). Exercise, fitness, and health: A consensus of current knowledge (pp. 33-48). Champaign, IL: Human Kinetics.

Papalia, D. E., Olds, S. W. \& Feldman, R. D. (2006). Desenvolvimento humano ( ${ }^{\mathrm{a}} \mathrm{ed}$.). Porto Alegre: Artmed.

Pelletier, L. G. (2002). A motivational analysis of selfdetermination for pro-environmental behaviors. Em E. L. Deci \& R. M. Ryan (Eds.). Handbook of self-determination research (pp. 205-232). Rochester, NY: University of Rochester Press.

Pelletier, L. G., Dion, S., Tuson, K. M. \& Green-Demers, I. (1999). Why do people fail to adopt environmental behaviors? Towards a taxonomy of environmental amotivation. Journal of Applied Social Psychology, 29, 24812504.

Pestana, M. H. \& Gageiro, J. G. (2003). Análise de dados para ciências sociais: a complementaridade do SPSS ( $3^{\mathrm{a}}$ ed.). Lisboa: Silabo. 
Petherick, C. M. \& Weigand, D. A. (2002). The relationship of dispositional goal orientations and perceived motivational climates on indices of motivation in male and female swimmers. International Journal of Sport Psychology, 33, 218-237.

Plant, R. \& Ryan, R. M. (1985). Intrinsic motivation and the effects of self-consciousness, self-awareness, and ego-involvement: An investigation of internally controlling styles. Journal of Personality, 53, 435-449.

Reeve, J., Deci, E. L. \& Ryan, R. M. (2004). Selfdetermination theory: A dialectical framework for understanding socio-cultural influences on student motivation. Em S. Van Etten \& M. Pressley (Eds.). Big theories revisited (pp. 31-60). Greenwich, CT: Information Age Press.

Reis, E. (2001). Estatística multivariada aplicada (2a ed.). Lisboa: Silabo.

Ryan, R. M. (1982). Control and information in the intrapersonal sphere: An extension of cognitive evaluation theory. Journal of Personality and Social Psychology, 43, 450-461.

Ryan, R. M. \& Deci, E. L. (2000a). Intrinsic and extrinsic motivations: Classic definitions and new directions. Contemporary Educational Psychology, 25(1), 54-67.

Ryan, R. M., \& Deci, E. L. (2000b). Self-Determination Theory and the facilitation of intrinsic motivation, social development, and well-being. American Psychologist, 55(1), 68-78.

Ryan, R. M. \& Lynch, M. F. (2003). Philosophies of motivation and classroom management. Em R. Curren (Ed.). Blackwell companions to philosophy: $A$ companion to the philosophy of education (pp. 260-271). Nova Iorque, NY: Blackwell.

Ryan, R. M., Mims., V. \& Koestner, R. (1983). Relation of reward contingency and interpersonal context to intrinsic motivation: A review and test using Cognitive Evaluation Theory. Journal of Personality and Social Psychology, 45, 736-770.

Ryan, R. M., Rigby, S. \& King, K. (1993). Two types of religious internalization and their relations to religious orientations and mental health. Journal of Personality and Social Psychology, 65, 586-596.

Ryan, R. M., Frederick, C. M., Lepes, D., Rubio, N. \& Sheldon, K. M. (1997). Intrinsic motivation and exercise adherence. International Journal of Sport Psychology, 28, 335-354.

Sheldon, K. M., Williams, G. C. \& Joiner, T. (2003). SelfDetermination Theory in the clinic: Motivating physical and mental health. New Haven, CT: Yale University Press.
Strahan, B. J. \& Craig, B. (1995). Marriage, family, and religion. Sydney, Australia: Adventist Institute of Family Relations.

Taylor, G. J., Bagby, R. M. \& Parker, J. D. A. (2003). The twenty $\tilde{n}$ Item Toronto Aleitheymia Scale IV. Reliability and factorial validity in different languages and cultures. Journal of Psychosomatic Research, 55, 277-283.

Trudel, R. \& Antonius, R. (1991). Métodes quantitatives appliquées aux sciences humaines. Montréal : Les Éditions de la Chenelière.

Vallerand, R. J. (1989). Vers une méthodologie de validation transculturelle des questionnaires psychologiques: implications pour la recherche en langue française. Psychologie Canadienne, 30, 662-680.

Vallerand, R. J. \& Losier, G. F. (1999). An integrative analysis of intrinsic and extrinsic motivation in sport. Journal of Applied Sport Psychology, 11, 142-169.

Vansteenkiste, M., Lens, W., Dewitte, S., De Witte, H. \& Deci, E. L. (2004). The "why" and "why not" of job search behaviour: Their relation to searching, unemployment experience, and well-being. European Journal of Spcial Psychology, 34, 345-363.

Villacorta, M., Koestner, R. \& Lekes, N. (2003). Further validation of the motivation toward the Environment Scale. Environment and Behavior, 35, 486-505.

Wankel, L. M. (1993). The importance of enjoyment to adherence and psychological benefits from physical activity. International Journal of Sport Psychology, 24, 151169.

Watkins D. (1989). The role of confirmatory factor analysis in cross-cultural research. International Journal of Psychology, 24, 685-701.

Williams, G. C. (2002). Improving patients' health through supporting the autonomy of patients and providers. Em E. L. Deci \& R. M. Ryan (Eds.). Handbook of self-determination research (pp. 233-254). Rochester, NY: University of Rochester Press.

Williams, G. C., Deci, E. L. \& Ryan, R. M. (1998). Building health-care partnerships by supporting autonomy: Promoting maintained behavior change and positive health outcomes. Em A. L. Suchman, P. Hinton-Walker \& R. Botelho (Eds.). Partnerships in healthcare: Transforming relational process (pp. 67-87). Rochester, NY: University of Rochester Press.

Recebido em maio de 2007 Reformulado em abril de 2008 Aprovado em abril de 2008 
Sobre os autores:

Marcos Alencar Abaide Balbinotti é PhD em Psicologia pela Universidade de Montreal e pós-doutor em variáveis afetivas (Universidade de Montreal, 2000-2001) e em desenvolvimento de carreira (Universidade de Sherbrooke, 2006-2008). Desenvolve estudos com variáveis relacionadas a escolha e desenvolvimento profissional, educação à carreira, psicologia do esporte e psicometria. É pesquisador do Centro de Pesquisas Interuniversitárias sobre a Educação e a Vida no Trabalho, órgão canadense; professor do Pós-Graduação em Estudos de Carreira na Universidade do Quebec em Montreal, pesquisador em licença dos Mestrados em Ciências da Saúde (Unisinos) e Ciências do Movimento Humano (UFRGS), coordenador do Núcleo de Orientação Vocacional da Universidade do Vale do Rio dos Sinos e professor associado ao Núcleo de Estudos e Pesquisas em Psicologia e Pedagogia do Esporte (NEPPPE/UFRGS).

Marcus Levi Lopes Barbosa é doutorando pelo Programa de Pós-Graduação em Ciências do Movimento Humano (PPGCMH-UFRGS) e mestre em Ciências do Movimento Humano pela Universidade Federal do Rio Grande do Sul (UFRGS). Desenvolve estudos em Motivação Humana, Variáveis Relacionadas à Motivação e Performance de Atletas, Psicometria. É pesquisador do Núcleo de Estudos e Pesquisas em Psicologia e Pedagogia do Esporte (NEPPPE/UFRGS). 